\title{
Analysis of RADAR Performance for Different Fluctuating Target Models of Various False Alarm Rate
}

\author{
Md. Maynul Islam ${ }^{1}$ and Mohammed Hossam-E-Haider ${ }^{2}$ \\ Department of Electrical, Electronic and Communication Engineering \\ Military Institute of Science and Technology, Mirpur Cantonment, Dhaka.
}

\section{Abstract}

By transmitting electromagnetic energy, a radar system can detect targets in various ranges. Radar return is referred to that energy which is reflected off targets within the search volume. An antenna receives the radar return plus noise. This signal is then processed to determine target characteristics such as range and velocity relative to the radar antenna. For any case if the sample magnitude of the radar return crosses the threshold voltage, then its probability is called the Probability of Detection, $\mathrm{P}_{\mathrm{d}}$. Radar cross section (RCS) can lower the probability of detection for a scatterer which have variations within the cross-section range. Swerling Fluctuating Target Models can describe the fluctuation of a scatterer by four specific cases. This paper has proposed a technique to increase the detection probability of radar for fluctuating targets under various false alarm rate.

Keywords - Swerling model, CFAR, Cell averaging, Coherent detection, Cell array.

\section{Introduction}

Swerling developed a mathematical methodology for calculating the detection of fluctuating targets. Five Swerling target models have been established which are special cases of Chi-Squared target models [1]. In Swerling I, RCS varies with two degrees of freedom according to a Chi-squared probability density function. Swerling I \& III treats a target whose radar cross-section is fixed throughout a single scan, but varies independently from scan to scan. But in Swerling II \& IV radar cross section varies from pulse to pulse and remain constant through scan to scan. The detection of signals becomes complex when radar returns from non-stationary background noise (or noise plus clutter). A radar target can be represented as a function of time on the basis of a large number of real targets whose return changes in magnitude from low to high. In Constant False Alarm Rate (CFAR), the estimation of the noise power levels from the leading andthe trailing reference windows are based on theCell Averaging (CA) technique [2-4]. The performance of this detector is analyzed in the cases when the operating environment is ideal and when it includes some of spurious targets along with the target of interest. The primary and the secondary interfering targets are assumed to be fluctuating in accordance with the four Swerling's models cited above. The theoretical results show that for various False Alarm rates the probability of detection will be different.

\section{System Model}

The probability of detection $\mathrm{P}_{\mathrm{D}}$ is the probability that a sample $\mathrm{R}$ of $\mathrm{r}(\mathrm{t})$ will exceed the threshold voltage in the case of noise plus signal,

$$
P_{D}=\int_{V_{T}}^{\infty} \frac{r}{\Psi^{2}} I_{o}\left(\frac{r A}{\Psi^{2}}\right) \exp \left(-\left(r^{2}+A^{2}\right) / 2 \Psi^{2}\right) d r
$$

If we assume that the radar signal is a sine waveform with amplitude $A$, then its power is $\mathrm{A}^{2} / 2$.

In many cases the radar detection threshold is constantly adjusted as a function of the receiver noise level in order to maintain a constant false alarm rate [5]. For this purpose, Constant False Alarm Rate (CFAR) processors are utilized in order to keep the number of false alarms under control in a 
changing and unknown background of interference. CFAR processing can cause a loss in the SNR level on the order of $1 \mathrm{~dB}$. The detection threshold is computed so that the radar receiver maintains a constant pre-determined probability of false alarm. A relationship between the threshold value $V_{T}$ and the probability of false alarm $\mathrm{P}_{\mathrm{fa}}$ can be defined as:

$V_{T}=\sqrt{2 \Psi^{2} \ln \left(\frac{1}{P_{f a}}\right)}$

If the noise power $\Psi^{2}$ is assumed to be constant, then a fixed threshold can satisfy the above equation. However, due to many reasons this condition is rarely true. In order to maintain a constant probability of false alarm the threshold value must be continuously updated based on the estimates of the noise variance. The process of continuously changing the threshold value to maintain a constant probability of false alarm is known as Constant False Alarm Rate (CFAR).

Cell averaging is performed on a series of range and Doppler bins (cells) [6]. The echo return for each pulse is detected by a square law detector. In analog implementation, these cells are obtained from a tapped delay line. The Cell Under Test (CUT) is the central cell. The immediate neighbors of the CUT are excluded from the averaging process due to possible spill over from the CUT. The output of $\mathrm{M}$ reference cells (M/2 on each side of the CUT) is averaged. The threshold value is obtained by multiplying the average estimate from all reference cells by a constant $\mathrm{K}_{\mathrm{O}}$ (used for scaling). A detection is declared in the CUT if

$Y_{1} \geq K_{o} Z$

Where $\mathrm{Y}_{1}$ is sum of squared envelopes mentioned in the figure below and $\mathrm{Z}$ is random variable.

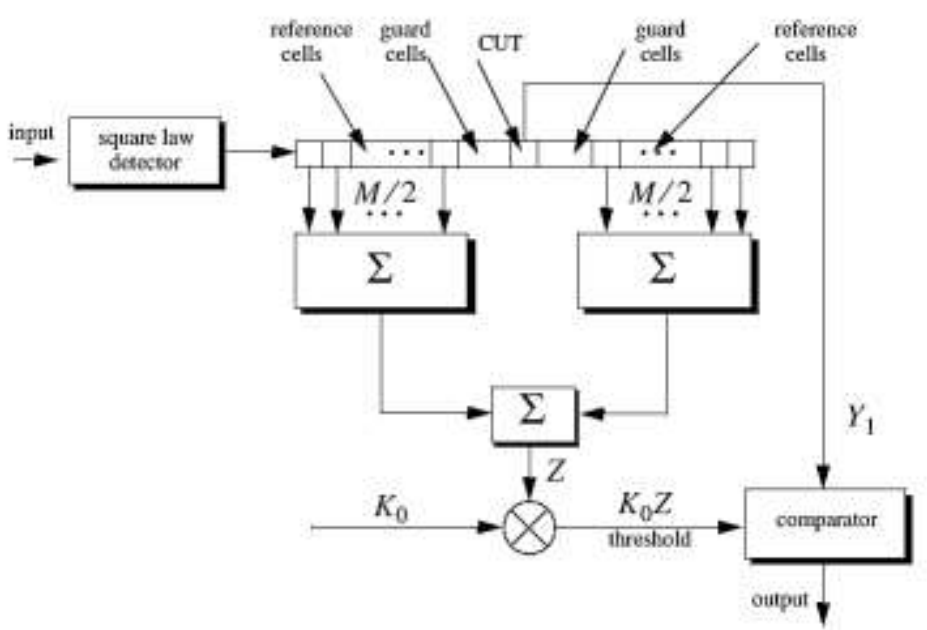

Fig. 1. Conventional CA-CFAR with coherent integration

The probability of false alarm corresponding to a fixed threshold was derived earlier. When CACFAR is implemented, then the probability of false alarm can be derived from the conditional false alarm probability, which is averaged over all possible values of the threshold in order to achieve an unconditional false alarm probability. The conditional probability of false alarm when $y=V_{T}$ can be written as

$P_{f a}\left(V_{T}\right)=e^{-\left(\frac{y}{2 \Psi^{2}}\right)}$

As a result, unconditional probability of false alarm is 
$P_{f a}=\int_{0}^{\infty} P_{f a}(y) f(y) d y$

Where, $f(y)$ is the $p d f$ of the threshold value.

Practically, CFAR averaging is often implemented after non-coherent integration and the output of each reference cell is the sum of squared envelopes. It follows that the total number of summed reference sample is $\mathrm{Mn}_{\mathrm{p}}$. The output $\mathrm{Y}_{1}$ is also the sum of $\mathrm{n}_{\mathrm{p}}$ squared envelopes. When noise alone is present in the CUT, $\mathrm{Y}_{1}$ is random variable whose $p d f$ is a gamma distribution with $2 \mathrm{n}_{\mathrm{p}}$ degrees of freedom. Additionally, the summed output of the reference cells is the sum of $\mathrm{Mn}_{\mathrm{p}}$ squared envelopes. Thus, $\mathrm{Z}$ is also a random variable which has a gamma $p d f$ with $2 \mathrm{Mn}_{\mathrm{p}}$ degrees of freedom [7-8]. The probability of false alarm is then equal to the probability that the ratio $\mathrm{Y}_{1} / \mathrm{Z}$ exceeds the threshold. More precisely,

$P_{f a}=\operatorname{Prob}\left\{\frac{Y_{1}}{Z}>K\right\}$

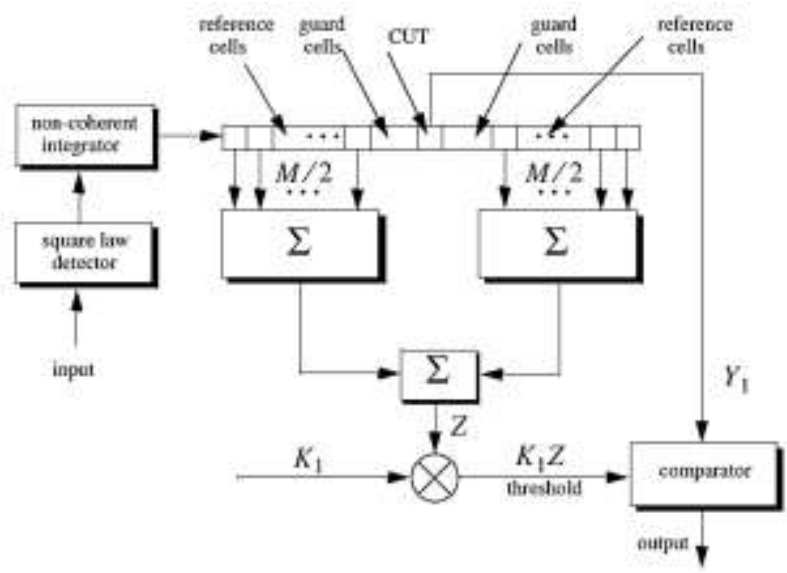

Fig. 2. Conventional CA-CFAR with non coherent integration

\section{Simulation}

Simulation has been carried out for different Swerling Model in terms of fluctuating target. Conventional Cell Averaging CFAR has been used here. For different false alarm rate the probability of detection will be different if the Signal to Noise Ratio (SNR dB) varies firmly.

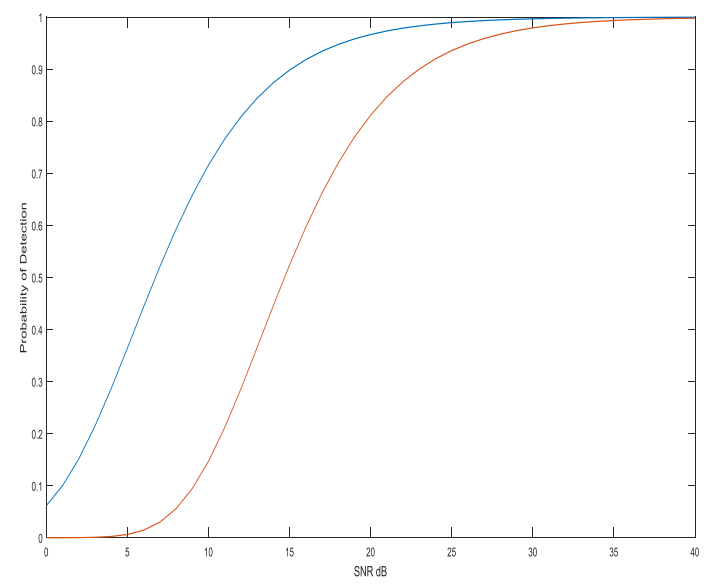

Fig. 3. Probability of Detection vs SNR(dB) for different no. of pulses 
Probability of detection and constant false alarm rate have been calculated using conventional formula. For every model of Swerling, $\mathrm{P}_{\mathrm{d}}$ and CFAR have simulated \& compared. At first detection probability of target detection was found out with respect to Signal to Noise Ratio (SNR). After that Constant false alarm rate was calculated and compared for different swerling model on fluctuating target.

\section{Result and Discussion}

In this paper, comparison has been created for $\mathrm{P}_{\mathrm{d}}$ vs CFAR for four types of Swerling model. From figure 4 it is clear that for different CFAR, the value of probability of detection is different. For simplicity we can assume the value of CFAR is 2 and 10 to determine probability of detection in each case of Swerling.

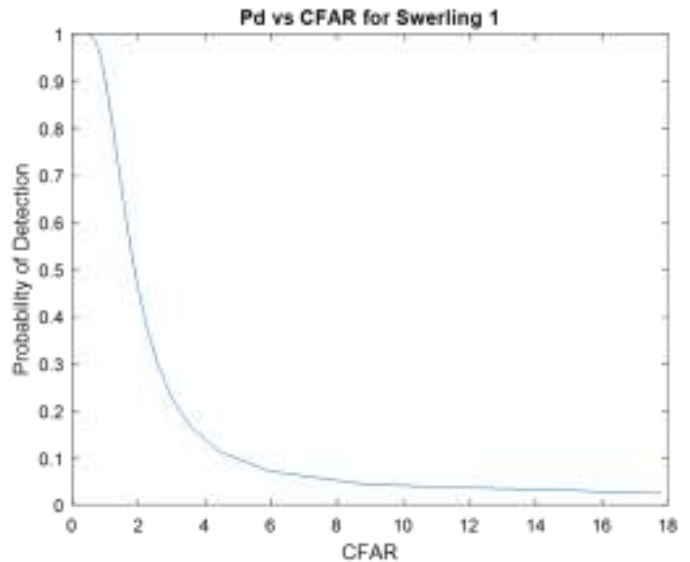

(a)

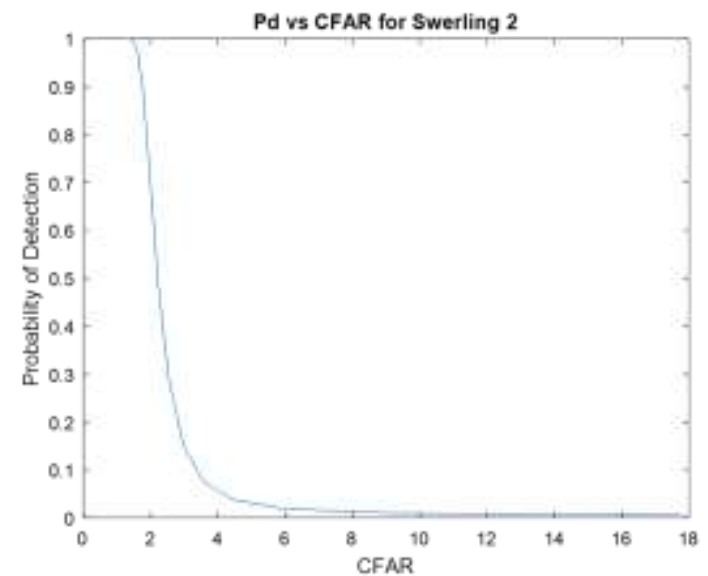

(b)

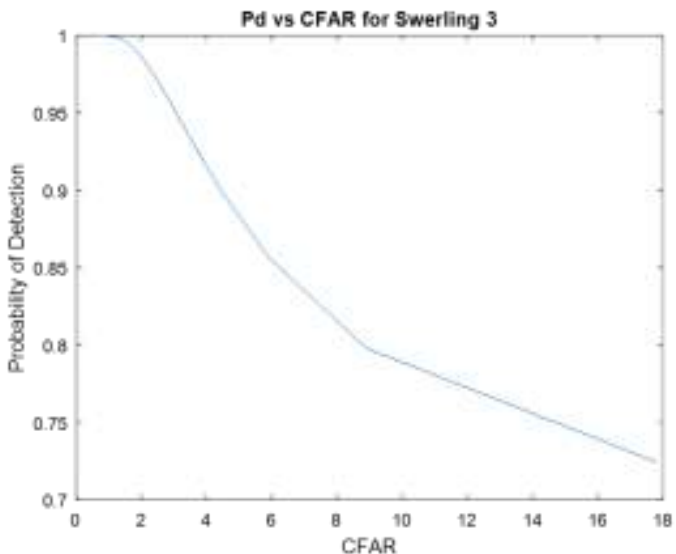

(c) 


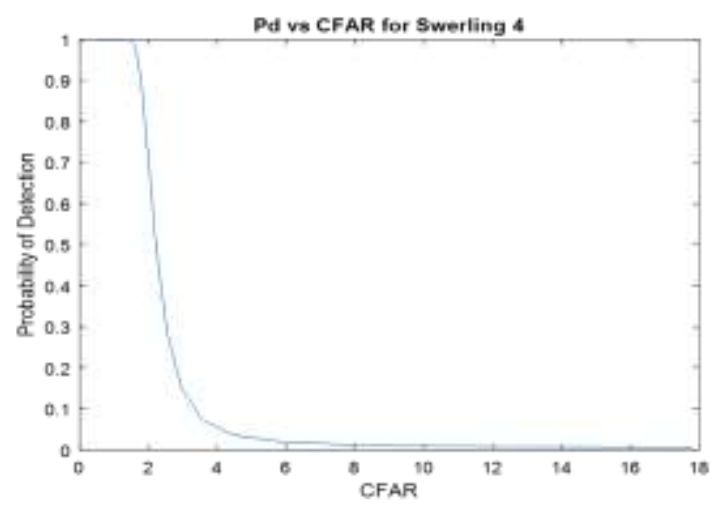

(d)

Fig. 4. Probability of Detection, $P_{d}$ Vs CFAR (a) Swerling I (b) Swerling II (c) Swerling III (d) Swerling IV

TABLE I: $P_{d}$ vs CFAR

\begin{tabular}{|c|l|l|c|c|}
\hline \multirow{2}{*}{ Swerling Model } & \multicolumn{2}{|c|}{ Observation A } & \multicolumn{2}{c|}{ Observation B } \\
\cline { 2 - 5 } & CFAR & \multicolumn{1}{|c|}{$\mathrm{P}_{\mathrm{d}}$} & CFAR & $\mathrm{P}_{\mathrm{d}}$ \\
\hline I & 2 & 0.46 & 10 & 0.04 \\
\hline II & 2 & 0.7 & 10 & 0.01 \\
\hline III & 2 & 0.98 & 10 & 0.77 \\
\hline IV & 2 & 0.6 & 10 & 0.01 \\
\hline
\end{tabular}

Two observations have been taken from the above table for two random values of CFAR. It is shown that for increasing rate of CFAR the probability of detection is decreasing. As a result, at low CFAR the value of $\mathrm{P}_{\mathrm{D}}$ will be maximum. If we consider a certain value of CFAR it is seen that for Swerling model III, the probability of detection is higher than any other model. It is clear that Swerling model II has the second highest value of $\mathrm{P}_{\mathrm{D}}$ in this comparison which pointed out in the above table.

\section{Conclusion}

This paper presents an analytical method for comparison of probability of detection for various CFAR in RADAR technology. Differences among four Swerling model have been experimentally demonstrated in the case of fluctuating target. It is observed that lower constant false alarm rate can give better accuracy in detection. It has been revealed that decreasing the value of CFAR as well as increasing Cell Array can be the better solution where targets are fluctuated. In future analysis, comparison between CFAR and Cell array can be an additional version of this research. Due to inherent nature of coherent and non coherent integration the proposed system is flexible, easy to implement.

\section{REFERENCES}

I. D. A. Shnidman, "Expanded Swerling target models," IEEE Transactions on Aerospace and Electronic Systems, vol. 39(3), pp. 1059 - 1069, Aug. 2003.

II. C. J. Willis, "Target modeling for SAR image simulation," The International Society for Optical Engineering, vol. 9243, Oct. 2014.

III. B. Thomas and S. L. Donnie, "Improved RCS model for censored Swerling III and IV target models," in IEEE Aerospace Conference Proceedings, 2013. 
IV. E. M. Mohamed, "Analytical performance evaluation of adaptive detection of fluctuating radar targets," Radioelectronics and Communications Systems, vol. 56(7), pp. 321-334, Jul. 2013.

V. K. Lingjiang, C. Guolong, Y. Xiaobo, and W. Bing, "Constant false alarm rate performance prediction for non-independent and non-identically distributed gamma fluctuating targets," IET Radar Sonar Navigation, vol. 10(5), Oct. 2015.

VI. W. Bing, C. Guolong, K. Lingjiang, and Y. Xiaobo, "Performance prediction of the CFAR detector for generalized Swerling-Chi fluctuating targets," in IEEE National Radar Conference Proceedings, 2013, vol. 52(1), pp. 1-5.

VII. I. Ilfiyantri, H. Rahmawati, H. Reza, and M. Achmad, "Improvement of Radar Performance Using LFM Pulse Compression Technique," in ICEEI Proceedings, 2015.

VIII. L. H., Z. S., and Y. J., "An integrated target detection and tracking algorithm with constant track false alarm rate," Journal of European Industrial Training, May 2016. 